\title{
ALGEBRAIC LIMIT CYCLES ON QUADRATIC POLYNOMIAL DIFFERENTIAL SYSTEMS
}

\author{
JAUME LLIBRE AND CLAUDIA VALLS
}

\begin{abstract}
Algebraic limit cycles in quadratic polynomial differential systems started to be studied in 1958, and few years later the following conjecture appeared: Quadratic polynomial differential systems have at most one algebraic limit cycle.

We prove that for a quadratic polynomial differential system having an invariant algebraic curve with at most one pair of diametrally opposite singular point at infinity, has at most one algebraic limit cycle. Our result provides a partial positive answer to this conjecture.
\end{abstract}

\section{INTRODUCTION}

Let $\mathbb{R}[x, y]$ be the ring of all real polynomials in the variables $x$ and $y$. Differential systems of the form

$$
\frac{d x}{d t}=\dot{x}=P(x, y), \quad \frac{d y}{d t}=\dot{y}=Q(x, y),
$$

where $P, Q \in \mathbb{R}[x, y]$ with $t$ real are called real polynomial differential systems. We say that system (1) has degree $m$ if the maximum degree of the polynomials $P$ and $Q$ is $m$. When $m=2$, system (1) is called a quadratic system.

The polynomial vector field associated to system (1) is

$$
\mathcal{X}=P(x, y) \frac{\partial}{\partial x}+Q(x, y) \frac{\partial}{\partial y} .
$$

Let $g=g(x, y) \in \mathbb{R}[x, y]$. The algebraic curve $g(x, y)=0$ of $\mathbb{R}^{2}$ is an invariant algebraic curve of the polynomial vector field $\mathcal{X}$ if for some polynomial $K \in \mathbb{R}[x, y]$, called the cofactor, we have

$$
\mathcal{X} g=P \frac{\partial g}{\partial x}+Q \frac{\partial g}{\partial y}=K g \text {. }
$$

Note that $g=0$ is invariant under the flow defined by $\mathcal{X}$.

An invariant algebraic curve $g=0$ where $g$ is irreducible in $\mathbb{R}[x, y]$ is called irreducible. A limit cycle of a real polynomial vector field $\mathcal{X}$ is an isolated periodic orbit in the set of all periodic orbits of $\mathcal{X}$. An algebraic limit cycle of degree $n$ of $\mathcal{X}$ is an oval of a real irreducible invariant algebraic curve $g=0$ of degree $n$ which is a limit cycle of $\mathcal{X}$.

A point $(x, y)$ of an algebraic curve $g=0$ is called a singular point of the curve if $g(x, y)=g_{x}(x, y)=g_{y}(x, y)=0$. In order to avoid confusions in

2010 Mathematics Subject Classification. Primary: 37D99.

Key words and phrases. algebraic limit cycle, quadratic polynomial differential system, quadratic polynomial vector field. 
this paper we will distinguish between a singular point of a curve and an equilibrium point of the system, i.e. a point such that $P(x, y)=Q(x, y)=0$. It is well-known that a singular point of an invariant algebraic curve must be an equilibrium point of the system. The converse in general does not hold.

The following problem is a simpler version of the second part of Hilbert's 16th problem (see [14]): Let $\Sigma_{m}$ be the set of all real polynomial vector fields (2) of degree $m$ having invariant algebraic curves. Is there a uniform upper bound on the number of algebraic limit cycles of any polynomial vector field of $\Sigma_{m}$ ?

In [17] the authors give a positive answer to this question when all the invariant algebraic curves $g_{j}=0$ of a vector field in $\Sigma_{m}$ satisfy the following assmptions: $g_{j}=0$ is a nonsingular algebraic curve, the highest order homogeneous terms of $g_{j}$ have no repeated factors, if two curves intersect at a point in the affine plane they are transversal at this point, there are no more than two curves $g_{j}=0$ meeting at any point in the affine plane, and there are no two curves having a common factor in the highest order homogeneous terms. For other related papers concering this problem, see [18] and [23]. It remains to know if when the invariant algebraic curves of a quadratic polynomial differential systems do not satisfy these generic conditions also have at most one algebraic limit cycle.

In [25] it is proved that quadratic polynomial vector fields can have algebraic limit cycles of degree 2, and that they are unique whenever they exist. In $[10,11,12]$ the author proved that quadratic vector fields do not have algebraic limit cycles of degree 3 (see also $[4,15]$ for different and shorter proofs). In [24] it is found the first class of algebraic limit cycles of degree 4 inside the quadratic vector fields. The second class was found in [13]. More recently, two new classes have been found and in [7] the authors proved that there are no other algebraic limit cycles of degree 4 for quadratic vector fields. The uniqueness of these limit cycles was proved in [2]. It is known that there are quadratic polynomial differential systems having algebraic limit cycles of degree 5 and 6 , see [7], and that this limit cycle is the unique one for these differential systems.

It turns out that the problem mentioned above is too hard to deal with and that is why a simpler version of this problem has kept the attention of the researchers for many years. It is the following conjecture which appears explicitly in $[16,18]$, but was known many years before among the mathematicians working in this subject.

Conjecture 1. Quadratic polynomial differential systems have at most one algebraic limit cycle.

In this paper we will prove this conjecture for the case in which the invariant algebraic curve has at most one pair of diametrally opposite singular points at infinity. We note that if an invariant algebraic curve has one singular point at infinity, then its diametrally opposite point at infinity is also singular. In [20] the authors proved the conjecture in the case in which the quadratic polynomial differential systems have only one pair of equilibrium points at infinity (which may not be singular) and in [?] the authors proved 
the conjecture in the case in which the quadratic polynomial differential systems have two pairs of equilibrium points at infinity (which may not be singular). In the following result we restrict ourselves in the case in which the system has three pairs of singular points at infinity.

Theorem 2. For a quadratic polynomial differential system with three pairs of equilibrium points at infinity having an invariant algebraic curve with at most no singular points at infinity, has at most one algebraic limit cycle.

Theorem 3. For a quadratic polynomial differential system with three pairs of equilibrium points at infinity having an invariant algebraic curve with at most one pair of diametrally opposite singular points at infinity, being the endpoints of the $y$-axis has at most one algebraic limit cycle.

We recall that an invariant algebraic curve $g=0$ of a quadratic system may have at most 4 finite singular points, and at most 3 pairs of infinite singular points (due to the fact that the singular points must be equilibrium points of the system). The known cases with algebraic limit cycles that are realized with invariant algebraic curves of degrees 5 or 6 are curves with singular points: they have one finite singular point (which is the origin) and one pair of infinite singular points. In the example of degree 6 the pair of infinite singular points are the endpoints of the $y$-axis. See [7] for the explicit expressions of the known algebraic limit cycles of degrees 5 and 6 .

In view of Theorem 3 it remains to prove the conjecture in the case that the system has three pairs of equilibrium points at infinity and some of its invariant algebraic curves have either a pair of diametrally opposite singular points which are not the endpoints of the $x$-axis or more than one pair of diametrally opposite singular points at infinity.

The proof of Theorem 2 is given in section 2 and the proof of Theorem 3 is given in section 3 . In section 2 we also state some known facts about quadratic polynomial differential systems that we shall need.

\section{Quadratic systems: Preliminary Results}

The following theorems are well known. For a proof of the first one see $[2,4]$.

Theorem 4. Quadratic polynomial differential systems having an algebraic limit cycle of degree two or four have at most one limit cycle.

In view of Theorem 4 from now on we will consider algebraic limit cycles of degree $n \geq 5$.

The next result is proved in $[8,9]$.

Theorem 5. Quadratic polynomial differential systems having an invariant algebraic straight line have at most one limit cycle.

Two results were proved in [19]. Proposition 8 of [19] states:

Proposition 6. All the equilibrium points of the quadratic polynomial differential system (4) and all points satisfying $\partial g / \partial x=\partial g / \partial y=0$ of an invariant algebraic curve $g=0$ are contained in the union of $\{K=0\}$ and $\{g=0\}$, where $K$ is the cofactor of $g=0$. 
From Theorem 4 of [19] and its proof and Theorem 2 of [20] we have:

Theorem 7. If a quadratic polynomial differential system has an algebraic limit cycle of degree $n$, then it can be transformed, through an affine change of variables and a scaling of the time, into one of the following two systems. First

$$
\begin{aligned}
& \dot{x}=\xi x-y+a x^{2}+b x y, \\
& \dot{y}=x-\xi y+d x^{2}+e x y+f y^{2},
\end{aligned}
$$

with $d \neq 0$ and second

$$
\begin{aligned}
& \dot{x}=-y+a x^{2}+b x y+c y^{2}, \\
& \dot{y}=x+e x y+f y^{2} .
\end{aligned}
$$

The cofactor of $g=0$ in both cases is ny.

It is pointed out in [19] that in system (4) the limit cycle, if it exists, it surrounds the origin.

Proposition 8. Let $P_{2}$ and $Q_{2}$ be the homogeneous components of $P$ and $Q$, respectively. If y $P_{2}-x Q_{2} \equiv 0$, then the quadratic system has no limit cycles.

The following result is Lemma 11 in [19].

Proposition 9. The invariant algebraic curve $g=0$ must intersect the infinity at least in one point, eventually complex. All the intersection points must be equilibrium points of the extended vector field in the projective space.

The following lemma due to Christopher [6] says how must be the higher degree terms of an invariant algebraic curve $g=0$ of a polynomial differential system (1).

Proposition 10. Suppose that a polynomial differential system (1) of degree 2 has the invariant algebraic curve $g=0$ of degree $n$. Let $P_{2}, Q_{2}$ and $g_{n}$ be the homogeneous components of $P, Q$ and $g$ of degree 2 and $n$ respectively. Then the irreducible factors of $g_{n}$ must be factors of $y P_{2}-x Q_{2}$.

The proof of the next theorem can be found in [6] (see also Theorem A2 in $[2])$.

Theorem 11 ([6]). The system

$$
\dot{x}=-y+a_{1} x+a_{2} x^{2}, \quad \dot{y}=x\left(1+a_{3} x+a_{4} y\right)
$$

has at most one limit cycle surrounding the origin.

The proof of the next theorem can be found in [20] (see Theorem 2).

Theorem 12. A quadratic polynomial differential system with at most one pair of equilibrium points at infinity, has at most one limit cycle.

In view of Theorem 12 in order to prove Theorem 3 we can restrict ourselves to the case in which the quadratic polynomial differential system has at least two pairs of equilibrium points at infinity.

The proof of the next theorem can be found in [?] (see Theorem 2).

Theorem 13. A quadratic polynomial differential system with two pairs of equilibrium points at infinity, has at most one limit cycle. 
In view of Theorem 13 in order to prove Theorem 3 we can restrict ourselves to the case in which the quadratic polynomial differential system has three pairs of equilibrium points at infinity.

2.1. Proof of Theorem 2. Let $g=0$ be an invariant algebraic curve of degree $n \geq 5$ of the quadratic polynomial differential systems (4). Then we can write $g=\left(\sum_{i=0}^{m} g_{n-i, i} x^{n-i} y^{i}\right)+\cdots, 0 \leq m \leq n$ with $g_{n-m, m} \neq 0$, where the dot denotes the terms of order $n-1$ and lower. From Proposition 10 we have

$$
\sum_{i=0}^{m} g_{n-i, i} x^{n-i} y^{i}=x^{n-m}\left(x-x_{1} y\right)^{k}\left(x-x_{2} y\right)^{m-k},
$$

where

$$
x_{1,2}=\frac{a-e \pm \sqrt{\Delta}}{2 d} \quad \text { with } \quad \Delta=(a-e)^{2}+4 d(b-f) \geq 0
$$

are the roots of the polynomial $d x^{2}-(a-e) x-(b-f)=0$. The case in which $\Delta \leq 0$ was proved in [20] and [?] because in that case at infinity there is at most two pairs of equilibrium points at infinity. Therefore $x_{1} \neq x_{2}$ and $x_{i} \neq 0$ for $i=1,2$. Note that in order that system (4) has no singular points at infinity we must have

$$
n-m \leq 1, k \leq 1, m-k \leq 1 \text { that is } m \leq k+1 \leq 2, n \leq m+1 \leq 3
$$

which is not possible because $n \geq 5$. Hence this case is not possible.

Let $g=0$ be an invariant algebraic curve of degree $n \geq 5$ of the quadratic polynomial differential systems (5). Then we can write $g=\left(\sum_{i=0}^{m} g_{n-i, i} y^{n-i} x^{i}\right)+$ ... with $g_{n-m, m} \neq 0$, where the dots indicate terms of degree $n-1$ and lower. By Propositions 8 and 10, we have that $\sum_{i=0}^{m} g_{n-i, i} y^{n-i} x^{i}=y^{n-m}(y-$ $\left.y_{1} x\right)^{k}\left(y-x_{2} x\right)^{m-k}$ with $0 \leq k \leq m$, where

$$
y_{1,2}=\frac{b-f \pm \sqrt{\Delta_{1}}}{2 d} \quad \text { with } \quad \Delta_{1}=(b-f)^{2}+4 c(a-e) \geq 0
$$

are the roots of the polynomial $c y^{2}+(b-f) y+(a-e)=0$. The case in which $\Delta \leq 0$ was proved in [20] and [?] because in that case at infinity there is at most two pairs of equilibrium points at infinity. Therefore $y_{1} \neq y_{2}$ and $y_{i} \neq 0$ for $i=1,2$. Note that in order that system (5) has no singular points at infinity we must have

$$
n-m \leq 1, k \leq 1, m-k \leq 1 \text { that is } m \leq k+1 \leq 2, n \leq m+1 \leq 3
$$

which is not possible because $n \geq 5$. Hence this case is not possible. This completes the proof.

\section{Proof of Theorem 3}

Let $g=0$ be an invariant algebraic curve of degree $n \geq 5$ of the quadratic polynomial differential systems (4). We write it as $g=\left(\sum_{i=0}^{m} g_{n-i, i} x^{n-i} y^{i}\right)+$ $\cdots, 0 \leq m \leq n$ with $g_{n-m, m} \neq 0$, where the dot denotes the terms of order 
$n-1$ and lower. The coefficient of the term $x^{n-m} y^{m+1}$ in the expression of $\dot{g}=n y g$ is equal to

$$
g_{n-m, m}((n-m) b+m f-n)=0 .
$$

Therefore

$$
b=(n-m f) /(n-m) \text { if } m \neq n \quad \text { and } \quad f=1 \text { if } m=n .
$$

From Proposition 10 we have that it can be written as in (6) and (7), i.e.

$$
\sum_{i=0}^{m} g_{n-i, i} x^{n-i} y^{i}=x^{n-m}\left(x-x_{1} y\right)^{k}\left(x-x_{2} y\right)^{m-k} .
$$

Note that the endpoint of $y=0$ is never a singular point of system (4) at infinity and so system (4) cannot have any singular point at infinity and thus this case is not possible.

So, we must assume that we have system (5). We will prove the following theorem.

Theorem 14. Let $g=0$ be an invariant algebraic curve with at most one singular point at infinity of the quadratic polynomial differential systems (5). Then system (5) has at most one limit cycle.

Proof. Let $g=\left(\sum_{i=0}^{m} g_{n-i, i} y^{n-i} x^{i}\right)+\cdots$ with $g_{n-m, m} \neq 0$, where the dots indicate terms of degree $n-1$ and lower. The coefficient of the term $y^{n-m} x^{m+1}$ in the expression $\dot{g}-n y g$ is equal to $a m+e(n-m)=0$. Therefore

$$
e=\frac{a m}{m-n} \text { if } m \neq n, \quad \text { and } \quad a=0 \text { if } m=n .
$$

By Propositions 8 and 10 (we are assuming that the line at infinity is not formed by equilibrium points, otherwise the system cannot have a limit cycle), we have that $\sum_{i=0}^{m} g_{n-i, i} y^{n-i} x^{i}=y^{n-m}\left(y-y_{1} x\right)^{k}\left(y-x_{2} x\right)^{m-k}$ with $0 \leq k \leq m$, where $y_{1}, y_{2}$ are the roots of $c y^{2}+(b-f) y+(a-e)=0$.

We now consider different cases: $m=n$ and $m<n$.

Case 1: $m=n$. In this case $a=0$. Proceeding as in [19], there are three reasons for having the condition $m=n$. First we simply have chosen the wrong system of coordinates, and there is some other real singular point of the system at infinity through which $g=0$ passes. In that case the system can be transformed into system (4) with $m<n$.

The second reason for having $m=n$ is that all the branches of $g=0$ go through non-real equilibrium points of the system at infinity. This means that $y_{1}=\overline{y_{2}} \notin \mathbb{R}$. In that case system (5) would have only a pair of equilibrium points at infinity and in view of Theorem 12 it is proved that in this case the system has at most one limit cycle.

The third reason for having $m=n$ is that $c=0$. Then system (5) becomes

$$
\dot{x}=-y+b x y, \quad \dot{y}=x+e x y+f y^{2} .
$$

If $b \neq 0$ system (5) has the invariant straight line $x=1 / b$ and in view of Proposition 5 system (5) has at most one limit cycle. So, $b=0$. Moreover, if $f=0$, then either the system has no equilibrium points at infinity (if $e \neq 0$ ) or the line at infinity is formed by equilibrium points (if $e=0$ ). In both 
cases it follows, respectively, from Propositions 9 and 8 that system (5) has no limit cycles.

In short, $b=0$ and $f \neq 0$. In this case $g_{n}=\left(y-\frac{e}{f} x\right)^{n}$. Imposing that

$$
\left(e x y+f y^{2}\right) \frac{\partial g_{n}}{\partial y}-n y g_{n}=0,
$$

we get

$$
y\left(y-\frac{e}{f} x\right)^{n-1}\left(\frac{e(f+1)}{f} x+(f-1) y\right)=0,
$$

and so

$$
f=1 \text { and } e=0 .
$$

It follows from Proposition 11 (making the change $x \rightarrow y$ and $y \rightarrow x$ ) that system (5) has at most one limit cycle.

Case 2: $m<n$. If $a=0$ then $e=0$ and it follows from Proposition 11 (making the change $x \rightarrow y$ and $y \rightarrow x$ ) that system (5) has at most one limit cycle.

We can thus assume that $a \neq 0$ and so $e=a m /(m-n)$. We distinguish between the cases $m=0$ (and then $k=0$ ), $m=1$ (with $k=0$ or $k=1$ ) and $m=2$ (with $k=1$ ). Note that if $c=0$ then we only have the cases $m=k=0$ and $m=1$ with $k=0$.

Subcase 2.1: $m=0$. In this case $g_{n}=y^{n}$ and imposing that $g_{n}$ satisfies

$$
\left(a x^{2}+b x y+c y^{2}\right) \frac{\partial g_{n}}{\partial x}+\left(\frac{a m}{m-n} x y+f y^{2}\right) \frac{\partial g_{n}}{\partial y}=n y g_{n}
$$

with $m=0$ we get $f=1$. We write $g$ in powers of $x$ as $g=\sum_{j=0}^{k} g_{j}(y) x^{j}$ where $g_{k} \neq 0$ (otherwise $g=0$ which is not possible). We obtain that the coefficient of $x^{k+1}$ satisfies $\operatorname{kag}_{k}(y)=0$. Since $a \neq 0$ then $k=0$ and so $g=g_{0}(y)$ and satisfies

$$
\left(x+y^{2}\right) \frac{d g_{0}}{d y}=n y g_{0} .
$$

The coefficient of $x$ satisfies $d g_{0} / d y=0$ and so $g_{0}$ is constant which is not possible becasue it is an invariant algebraic curve. Therefore, this case is not possible.

Subcase 2.2: $m=1$. We consider two different subcases $c=0$ and $c \neq 0$.

Subcase 2.2.1: $c=0$. We have that $g_{n}=y^{n-1}\left(y+\frac{a n}{(n-1)(b-f)} x\right)$. Imposing that $g_{n}$ satisfies (8) we get

$$
\frac{(f-1) n}{(b-f)(n-1)} y^{n}(a n x+(f-b+b n-f n) y)=0
$$

which yields, in particular, that $a=0$ and this is not possible.

Subcase 2.2.2: $c \neq 0$. We consider two different subcases: $k=0$ and $k=1$. In both cases $e=a /(1-n)$ and system (5) becomes

$$
\begin{aligned}
& \dot{x}=-y+a x^{2}+b x y+c y^{2}, \\
& \dot{y}=x+\frac{a}{1-n} x y+f y^{2} .
\end{aligned}
$$


We have $g_{n}=y^{n-1}\left(y+\frac{(b-f) \pm \sqrt{b^{2}-4 a c-2 b f+f^{2}+4 a c /(1-n)}}{2 c} x\right)(+$ for $k=0$ and - for $k=1$ ). Imposing that $g_{n}$ satisfies (8) we get

$$
\begin{aligned}
& \frac{1}{2 c(n-1)} y^{n}\left(\left( \pm(n-1)(b-f-n+f n) \sqrt{b^{2}-4 a c-2 b f+f^{2}+4 a c /(1-n)}\right.\right. \\
& -b^{2}+2 b f-f^{2}+b n+b^{2} n-2 a c n-f n-3 b f n+2 f^{2} n-b n^{2}+f n^{2} \\
& \left.+b f n^{2}-f^{2} n^{2}\right) x \pm c(n-1)\left(\sqrt{b^{2}-4 a c-2 b f+f^{2}+4 a c /(1-n)}+b\right. \\
& -f-2 n+2 f n) y)=0
\end{aligned}
$$

and so either $c=0$, or $a=0, b=f+n-f n$, or $c=(f-1)(1-n)(b-f-$ $n+f n) / a$. Since $a c \neq 0$ we must have $c=(f-1)(1-n)(b-f-n+f n) / a$.

Assume system (9) has a Darboux polynomial $g$ with cofactor $K=n y$. Then writing $g$ in powers of $x$ as $g=\sum_{j=0}^{k} g_{j}(y) x^{j}$ we obtain that the coefficient of $x^{k+1}$ satisfies

$$
\operatorname{kag}_{k}(y)+\left(1+\frac{a}{1-n} y\right) \frac{d g_{k}}{d y}=0
$$

and so

$$
g_{k}(y)=c_{k}(1-n+a y)^{(k(n-1)}
$$

Therefore

$$
g=c_{k} x^{k}(1-n+a y)^{k(n-1)}+\text { l.o.t }
$$

where l.o.t means terms of lower order in the variable $x$. Since the degree of $g$ is $n$ we must have that

$$
k+k(n-1) \leq n \quad \text { which implies } \quad k \leq 1 .
$$

Then

$$
g=x g_{1}(y)+g_{0}(y), \quad g_{1}(y)=c_{1}(1-n+a y)^{n-1}, \quad c_{1} \in \mathbb{R} .
$$

Moreover computing the terms of $x$, and the independent term we get that $g_{0}(y)$ must satisfy

$$
\begin{aligned}
& \text { byg }_{1}+f y^{2} g_{1}^{\prime}(y)+\left(1+\frac{a}{1-n} y\right) g_{0}^{\prime}(y)=n y g_{1}(y), \\
& \left(\frac{(f-1)(1-n)(b-f-n+f n)}{a} y^{2}-y\right) g_{1}(y)+f y^{2} g_{0}^{\prime}(y)=n y g_{0}(y) .
\end{aligned}
$$

We have

$$
\begin{aligned}
g_{0}(y) & =c_{0}+\frac{c_{1}(n-1)}{a^{2} n(n-2)}(1-n+a y)^{n-2}(-b(-2+n)(-1+n-a y) \\
& (1+a y)+(-2+n) n(-1+n-a y)(1+a y)+f(-1+n) \\
& \left.\left(-2(1+a y)^{2}+n\left(2+2 a y+a^{2} y^{2}\right)\right)\right) .
\end{aligned}
$$


From the third relation we get

$$
\begin{aligned}
& -c_{0} n y+\frac{c_{1}}{a^{2}(2-n)} y(1-n+a y)^{n-3}\left(-2 a^{2}+2 b-2 f-2 n+5 a^{2} n-7 b n\right. \\
& +8 f n+7 n^{2}-4 a^{2} n^{2}+9 b n^{2}-12 f n^{2}-9 n^{3}+a^{2} n^{3}-5 b n^{3}+8 f n^{3}+5 n^{4} \\
& +b n^{4}-2 f n^{4}-n^{5}+\left(-4 a^{3}+4 a b-4 a f+2 a b f-2 a f^{2}-10 a b n+9 a f n\right. \\
& -7 a b f n+9 a f^{2} n+10 a n^{2}-2 a^{3} n^{2}+8 a b n^{2}-a f n^{2}+9 a b f n^{2}-16 a f^{2} n^{2} \\
& -8 a n^{3}-2 a b n^{3}-11 a f n^{3}-5 a b f n^{3}+14 a f^{2} n^{3}+2 a n^{4}+9 a f n^{4}+a b f n^{4} \\
& \left.-6 a f^{2} n^{4}-2 a f n^{5}+a f^{2} n^{5}\right) y+\left(-2 a^{4}+2 a^{2} b-2 a^{2} f+2 a^{2} b f-4 a^{2} f^{2}\right. \\
& -2 a^{2} n+a^{4} n-3 a^{2} b n+a^{2} f n-5 a^{2} b f n+14 a^{2} f^{2} n+3 a^{2} n^{2}-4 a^{4} n^{2} \\
& +a^{2} b n^{2}+6 a^{2} f n^{2}+4 a^{2} b f n^{2}-18 a^{2} f^{2} n^{2}-a^{2} n^{3}-7 a^{2} f n^{3}-a^{2} b f n^{3} \\
& \left.\left.+10 a^{2} f^{2} n^{3}+2 a^{2} f n^{4}-2 a^{2} f^{2} n^{4}\right) y^{2}\right)=0 .
\end{aligned}
$$

Solving it we obtain that either $a=0, b=\left(n^{2}+2 f n-2 n-2 f\right) /(n-2)$, or $a= \pm \sqrt{b-n-b n+n^{2}}, f=0$. The first case is not possible, for the second and third cases we have that $f=0$ and so since $e \neq 0$ (because $a \neq 0$ ) we have that $y=-1 / e$ is an invariant straight line and in view of Proposition 5 , system (5) has at most one limit cycle. This completes the proof in this case.

Subcase 2.3: $m=2$ and $k=1$. We recall that $c \neq 0$ and $g_{n}=y^{n-2}\left(y^{2}+\right.$ $\left.\frac{a n x^{2}}{c(n-2)} x y+y^{2}\right)$. Imposing that $g_{n}$ satisfies (8) we get

$$
\frac{(b-f-n+f n) y^{n-1}}{c(n-2)}\left(a n x^{2}+(2 f-2 b+b n-f n) x y+c(n-2) y^{2}\right)=0
$$

which yields $b=f(1-n)+n$ because $a c \neq 0$.

Then $e=2 a /(2-n), b=n+(1-n) f, f \neq 0$ (because $m \neq 0)$ and system (5) becomes

$$
\begin{aligned}
& \dot{x}=-y+a x^{2}+(n+f(1-n)) x y+c y^{2}, \\
& \dot{y}=x+\frac{2 a}{2-n} x y+f y^{2} .
\end{aligned}
$$

Assume system (10) has a Darboux polynomial $g$ with cofactor $K=n y$. Then writing $g$ in powers of $x$ as $g=\sum_{j=0}^{k} g_{j}(y) x^{j}$ we obtain that the coefficient of $x^{k+1}$ satisfies

$$
\operatorname{kag}_{k}(y)+\left(1+\frac{2 a}{2-n} y\right) \frac{d g_{k}}{d y}=0
$$

and so

$$
g_{k}(y)=c_{k}\left(1+\frac{2 a}{2-n} y\right)^{k(n-2) / 2}
$$

Therefore

$$
g=c_{k} x^{k}\left(1+\frac{2 a}{2-n} y\right)^{k(n-2) / 2}+\text { l.o.t }
$$


where l.o.t means terms of lower order in the variable $x$. Since the degree of $g$ is $\mathrm{n}$ we must have that

$$
k+\frac{k(n-2)}{2} \leq n \quad \text { which implies } \quad k \leq 2 .
$$

Then

$$
g=x^{2} g_{2}(y)+x_{1} g_{1}(y)+g_{0}(y)
$$

with $g_{2}(y)=c_{2}\left(1+\frac{2 a}{2-n} y\right)^{n-2}$, where $c_{2} \in \mathbb{R}$. Moreover computing the terms of $x^{2}, x$ and the independent term we get that $f_{1}$ and $f_{0}$ must satisfy

$$
\begin{aligned}
& a g_{1}(y)+2(n+(1-n) f) y g_{2}(y)+\left(1+\frac{2 a}{2-n} y\right) \frac{d g_{1}}{d y}+f y^{2} \frac{d g_{2}}{d y}=n y g_{2}, \\
& (n+(1-n) f) y g_{1}(y)+2\left(c y^{2}-y\right) g_{2}+\left(1+\frac{2 a}{2-n} y\right) \frac{d g_{0}}{d y}+f y^{2} \frac{d g_{1}}{d y}=n y g_{1}, \\
& \left(c y^{2}-y\right) g_{1}(y)+f y^{2} \frac{d g_{0}}{d y}=n y g_{0} .
\end{aligned}
$$

Solving the first equation in (11) we get

$$
\begin{aligned}
g_{1}(y)= & c_{1}(2-n+2 a y)^{n / 2-1}+\frac{c_{2}(n-2)}{a^{2}(n-4)}(2-n+2 a y)^{n-3}((4+2 f(n-3) \\
& \left.-n)(n-2)+a(4+2 f(n-3)-n)(n-4) y-2 a^{2}(f-1)(n-4) y^{2}\right),
\end{aligned}
$$

with $c_{1} \in \mathbb{R}$. and solving the second equation in (11) we obtain

$$
\begin{aligned}
g_{0}= & \frac{n-2}{a^{2}(n-4)}\left(\frac { c _ { 1 } } { 4 } \left(\left(\frac{f}{n}\left(14 n-8-3 n^{2}\right)+2(n-4)\right)(2-n+2 a y)^{n / 2}\right.\right. \\
& \left.+f(n-2)^{3}(2-n+2 a y)^{n / 2-2}-2(f-1) n(n-4)(2-n+2 a y)^{n / 2-1}\right) \\
& +\frac{c_{2}}{8 a^{2}}\left(\frac{2 a c+(f-1)(f(n-1)-n)(n-2)(n-4)}{n}(2-n+2 a y)^{n}\right. \\
& +\frac{f^{2}(n-3)(n-2)^{6}}{n-4}(2-n+2 a y)^{n-4}+\frac{2 f(n-2)^{4}\left(8 f-3(f+1) n+n^{2}\right)}{n-3} \\
& (2-n+2 a y)^{n-3}+\left(-4 a^{2}(n-4)+2 a c(8+n(n-6))-(n-2)\left(-(n-4) n^{2}\right.\right. \\
& \left.\left.+f(8+n(n(15-2 n)-34))+2 f^{2}(6+n(n(n-5)+5))\right)\right)(2-n+2 a y)^{n-2} \\
& \left.-\frac{2(n-4)}{n-1}\left(2 a^{2}-2 a c(n-2)-(f-1)(f+1-n)(n-2) n\right)(2-n+2 a y)^{n-1}\right) \\
& +c_{0},
\end{aligned}
$$

with $c_{0} \in \mathbb{R}$.

We write the last relation in (11) as

$$
\mathcal{A}(y):=\left(c y^{2}-y\right) g_{1}(y)+f y^{2} \frac{d g_{0}}{d y}-n y g_{0}=0 .
$$

Note that $\mathcal{A}(y)$ is a polynomial in the variable $y$. Computing in $\mathcal{A}(y)=0$ the coefficient of the highest order term in $y$ (which is $y^{n+1}$ ) we get

$$
2^{n-3} a^{n-4} c_{2}(f-1)^{2}(n-2)^{2}((n-1) f-n)=0 .
$$


Since $a(n-2) \neq 0$ we have three possibilities: either $c_{2}=0$, or $f=1$, or $f=n /(n-1)$. We consider the three subcases separately.

Subcase 2.3.1: $c_{2}=0$. In this case

$$
\begin{aligned}
\left.\mathcal{A}(y)\right|_{c_{2}=0}= & -c_{0} n y-\frac{c_{1}}{a^{2}(n-4)} y(2-n+2 a y)^{n / 2-3}\left(-a^{3}(n-4)\left(-3 n^{2} f^{2}\right.\right. \\
& \left.+8 n f^{2}-4 f^{2}+8 n^{2} f-20 n f+8 f-4 n^{2}+4 a c+8 n\right) y^{3} \\
& +2 a^{2}(n-4)\left(-f^{2} n^{3}+4 f n^{3}-2 n^{3}+5 f^{2} n^{2}-21 f n^{2}+10 n^{2}\right. \\
& \left.-8 f^{2} n+2 a c n+32 f n-12 n+2 a^{2}+4 f^{2}-4 a c-12 f\right) y^{2} \\
& -a(n-2)\left(2 f n^{4}-n^{4}-24 f n^{3}+12 n^{3}+a c n^{2}+92 f n^{2}-44 n^{2}\right. \\
& \left.+4 a^{2} n-6 a c n-128 f n+48 n-16 a^{2}+8 a c+48 f\right) y \\
& +(n-2)^{2}\left(-2 f n^{3}+n^{3}+12 f n^{2}-6 n^{2}+a^{2} n-20 f n+8 n\right. \\
& \left.\left.-4 a^{2}+8 f\right)\right) .
\end{aligned}
$$

Note that $n$ must be even and $n \geq 5$, so $n \geq 6$. Then we compute the coefficients of $y^{2}, y^{3}, y^{4}, \ldots$ for $n>6$ and the coefficients of $y, y^{2}, y^{3}, y^{4}$ when $n=6$. Equating to zero the set of these coefficients and using that $f \neq 0$ and $c, a$ are real, we obtain that this system has no solution. So this subcase is not possible.

Subcase 2.3.2: $c_{2} \neq 0$ and $f=1$. In this case

$$
\begin{aligned}
\left.\mathcal{A}(y)\right|_{f=1}= & -c_{0} n y-\frac{c_{2}(n-3)(n-2)^{7} n}{8 a^{4}(n-4)^{2}} y(2-n+2 a)^{n-4}+\frac{c_{2}(n-2)^{5} n}{4 a^{4}(n-4)(n-3)} \\
& \left(-8+6 n-n^{2}\right) y(2-n+2 a)^{n-3}+\frac{c_{2} n(n-2)}{8 a^{4}(n-4)}\left(-40-16 a^{2}-16 a c\right. \\
& \left.+68 n+4 a^{2} n+12 a c n-42 n^{2}-2 a c n^{2}+11 n^{3}-n^{4}\right) y(2-n+2 a)^{n-2} \\
& +\frac{c_{2}(n-2) n(a+2 c-c n)}{2 a^{3}(n-1)} y(2-n+2 a)^{n-1}-\frac{c c_{2}(n-2)}{4 a^{3}} \\
& y(2-n+2 a)^{n}+c_{1}(-1+c y) y(2-n+2 a)^{n / 2-1}+c_{1}(n-2)(4(1-n) \\
& \left.+n^{2}+(2-n) a y\right) y^{3}(2-n+2 a)^{n / 2-3}-\frac{c_{2}(n-2)(-1+c y)}{a^{2}(n-4)} \\
& \left(4(n-1)-n^{2}+(6 n-8) a y-a n^{2} y\right) y(2-n+2 a)^{n-3}+\frac{c_{1}(n-2)}{a^{2}(n-4)} \\
& \left(8-12 n+6 n^{2}-n^{3}+a\left(16-20 n+8 n^{2}-n^{3}\right) y+a^{2}\left(8-6 n+n^{2}\right) y^{2}\right) \\
& y(2-n+2 a)^{n / 2-2}+\frac{c_{2}(n-2)}{a^{2}(n-4)}\left(32\left(a^{2}-1\right)+80 n-40 a^{2} n-80 n^{2}\right. \\
& +16 a^{2} n^{2}+40 n^{3}-2 a^{2} n^{3}-10 n^{4}+n^{5}+\left(-80 a+64 a^{3}-32 a^{2} c\right. \\
& +176 a n-48 a^{3} n+40 a^{2} c n-152 a n^{2}+8 a^{3} n^{2}-16 a^{2} c n^{2}+64 a n^{3} \\
& \left.+2 a^{2} c n^{3}-13 a n^{4}+a n^{5}\right) y+8 a^{2}\left(4 a^{2}-8 a c-a^{2} n+6 a c n-a c n^{2}\right) y^{2} \\
& \left.+8 a^{4} c(n-4) y^{3}\right) y^{3}(2-n+2 a)^{n-5} .
\end{aligned}
$$


Computing the coefficient of the highest power in $y$ which is $y^{n}$ we get

$$
\frac{2^{n-2} c_{2}(n-2)\left(a+4 c(1-n)+c n^{2}\right) a^{n-4}}{n-1}=0 .
$$

Taking into account that $c_{2}(n-2) a \neq 0$ we must have

$$
a=-c(n-2)^{2} .
$$

Now computing the coefficient of $y^{n-1}$ we obtain

$$
-\frac{2^{n-1} c_{2}^{n+1}(n-2)^{n-4}(n-5 / 2)}{c^{3}(n-3)}=0
$$

and this is not possible. So this case is not possible.

Subcase 2.3.3: $c_{2} \neq 0$ and $f=n /(n-1)$. In this case $\left.\mathcal{A}(y)\right|_{f=n /(n-1)}$ is very large and then we only write the coefficient of its highest power in $y$, which is $y^{n}$ and we get $2^{n-2} c_{2}(n-3)(-1)^{n}(n-2)^{2 n-7} c^{n-3}$ which is never zero. So this subcase is not possible. This completes the proof of Case 2 and concludes the proof of Theorem 14 .

The proof of Theorem 3 for system (5) follows directly from Theorem 14.

\section{ACKNOWLEDGEMENTS}

The first author is partially supported by a MINECO grant MTM201340998-P, an AGAUR grant number 2014SGR-568 and the grants FP7-PEOPLE2012-IRSES 318999 and 316338. The second author is partially supported by FCT/Portugal through the project UID/MAT/04459/2013.

\section{REFERENCES}

[1] M. Abramowitz And I.A. Stegun, Handbook of mathematical functions with formulas, graphs and mathematical tables, National Bureau of Standards Appl. Math. Series 55, 1964

[2] J. Chavarriga, H. Giacomini and J. Llibre, Uniqueness of algebraic limit cycles for quadratic systems, J. Math. Anal. Appl., 261 (2001), 85-99.

[3] J. ChavarRiga AND J. Llibre, Invariant algebraic curves and rational first integrals planar polynomial vector fields, J. Differential Equations 169 (2001), 1-16.

[4] J. Chavarriga, J. Llibre and J. Sorolla, Algebraic limit cycles of degree four for quadratic systems, J. Differential Equations 2009 (2004), 206-244.

[5] L.S. Chen, Acta Mathematica Sinica, (in chinese) 20 (1977), 11-13.

[6] C. Christopher, Invariant algebraic curves and conditions for a center, Proc. Roy. Soc. Edinburhgh. 124A (1994), 1209-1229.

[7] C. Christopher, J. Llibre And G. Swirszcz, Invariant algebraic curves of large degree for quadratic systems, J. Math. Anal. Appl. 303 (2005), 206-244.

[8] B. COLL AND J. LliBRE, Limit cycles for a quadratic systems with an invariant straight line and some evolution of phase portraits in Qualitative Theory of Differential Equations, Colloquia Mathematica Societatis Janos Bolyai, 53, Bolyai Institute, Hungary, 1988, 111-123.

[2] B. Coll, G. Gasull And J. Llibre, Quadratic systems with a unique finite rest point Publicacions Matematiques 32 (1988), 199-259

[9] W.A. Coppel, Some quadratic systems with at most one limit cycle Dynamics Reported, Vol. 2, 1989, 61-88.

[10] R.M. Evdokimenco, Construction of algebraic paths and the qualitative investigation in the large of the properties of integral curves of a system of differential equations, Differential Equations 6 (1970), 1349-1358. 
[11] R.M. Evdokimenco, Behavior of integral curves of a dynamic system, Differential Equations 9 (1974), 1095-1103.

[12] R.M. Evdokimenco, Investigation in the large of a dynamic systems with a given integral curve, Differential Equations 15 (1979), 215-221.

[13] V.F. Filiptsov, Algebraic limit cycles, Differential Equations 9 (1973), 983-986.

[14] D. Hilbert, Mathematische Probleme, in: Lecture, Second Internat. Congr. Math., Paris, 1900, in: Nachr. Ges. Wiss. Göttingen Math.-Phys. Kl., 1900, pp. 253?297; English transl. in: Bull. Amer. Math. Soc. 8 (1902) 4370-479.

[15] J. LLIBRE, Integrability of polynomial differential systems, Handbook of Differential Equations, Ordinary Differential Equations, Eds. A. Cañada, P. Drabek and A. Fonda, Elsevier, Vol. 1, 2004, pp 437-533.

[16] J. LliBRE, Open problems on the algebraic limit cycles of planar polynomial vector fields, Bull. of Academy of Sciences of Moldova (Matematica), vol. 56 (2008), 19-26.

[17] J. Llibre, R. Ramírez and N. Sadovskaia, On the 16th Hilbert problem for algebraic limit cycles, J. Differential Equations 248 (2010), 1401-1409.

[18] J. Llibre, R. Ramírez And N. Sadovskaia, On the 16th Hilbert problem for limit cycles on nonsingular algebraic curves, J. Differential Equations 250 (2011), 983-999.

[19] J. Llibre AND G. Swirszcz, Classification of quadratic systems admitting the existence of an algebraic limit cycle, Bulletin des Sciences Mathemàtiques 131 (2007), $405-421$.

[20] J. LliBRE AND C. VALLS, Quadratic polynomial differential systems with one pair of singular points at infinity have at most one algebraic limit cycle, preprint.

[21] B. SHEn, The problem of the existence of limit cycles and separatrix cycles of cubic curves in quadratic systems, (Chinese) Chinese Ann. Math. Ser. A 12 (1991), no. 3, $382-389$.

[22] X. ZHANG, Invariant algebraic curves and rational first integrals of holomorphic foliations in CP(2), Sci. China Ser. A 46 (2003), no. 2, 271-279.

[23] X. Zhang, The 16th Hilbert problem on algebraic limit cycles, J. Differential Equations 251 (2011), 1778-1789.

[24] A.I. YABLONSKII, Limit cycles of a certain differential equations, Differential Equations 2 (1966), 193-239.

[25] Quin Yuan-Xun, On the algebraic limit cycles of sencod degree of the differential equation $d y / d x=\sum_{0 \leq i+j \leq 2} a_{i j} x^{i} y^{j} / \sum_{0 \leq i+j \leq 2} b_{i j} x^{i} y^{j}$, Acta Math. Sinica 8 (1958), 23-35.

${ }^{1}$ Departament de Matemàtiques, Universitat Autònoma de Barcelona, 08193

Bellaterra, Barcelona, Catalonia, Spain

E-mail address: jllibre@mat.uab.cat

Departamento de Matemática, Instituto Superior Técnico, Universidade de Lisboa, 1049-001 LisboA, Portugal

E-mail address: cvalls@math.ist.utl.pt 